\title{
Éditorial
}

\section{Mobiliser les données probantes pour une meilleure action : de Maladies chroniques et blessures au Canada à Promotion de la santé et prévention des maladies chroniques}

\author{
Kerry Robinson, Ph. D., éditrice, Promotion de la santé et prévention des maladies chroniques au Canada \\ Michelle Tracy, M.A., gestionnaire de la rédaction, Promotion de la santé et prévention des maladies chroniques au \\ Canada
}

La revue Promotion de la santé et prévention des maladies chroniques au Canada: recherche, politique et pratique (PSPMC) (jusqu'à présent Maladies chroniques et blessures au Canada $[M C B C]$ ) est née à Santé Canada en 1980 dans la discrétion, sous forme d'un " nouveau bulletin " ayant pour objet de publier « des articles concernant les maladies non transmissibles, comme le cancer et les maladies cardiaques, et d'autres causes de morbidité et de mortalité, comme les accidents, en tenant compte de divers aspects comme la recherche, la surveillance et la lutte ${ }^{1}$. L'auditoire principal de cette nouvelle publication était constitué des quelque 300 à 400 professionnels canadiens participant directement ou indirectement à des programmes en lien avec les maladies chroniques.

Aujourd'hui, 35 ans plus tard, avec un facteur d'incidence de 1,22 , la revue est devenue une source fiable en matière de recherche évaluée par les pairs et une plateforme importante d'échanges de connaissances au sein de la communauté de la santé publique au Canada. Revue en libre accès et bilingue, PSPMC a également des lecteurs aux États-Unis, en Europe et en Afrique francophone. La revue a publié des centaines d'articles sur un éventail de sujets allant de la santé maternelle aux blessures et aux tendances en matière de cancer. Elle jouit d'une forte présence en ligne grâce à de nombreux index et agrégateurs de publications, notamment MEDLINE, Thomson Reuters, Elsevier, Scopus et EBSCO.
De la même manière que l'éventail des sujets traités dans la revue s'est élargi avec le temps et qu'on est passé d'une petite presse à imprimer à une publication entièrement accessible en ligne, la revue modifie maintenant son modèle de gouvernance et de production. Ce nouveau modèle reprend les pratiques de gouvernance appliquées à d'autres revues publiées par le gouvernement du Canada, par exemple Rapports sur la santé de Statistique Canada ou Examen nucléaire d'EACL (Énergie atomique du Canada limitée). En tant que publication relevant du gouvernement fédéral, la revue PSPMC va offrir des articles en science et en recherche appliquées à la prévention des maladies, à la promotion de la santé et à l'équité en santé dans les domaines des maladies chroniques, des blessures et de la santé à toutes les étapes de la vie, en mettant principalement l'accent sur la recherche et les collaborations de l'Agence de la santé publique du Canada. Toutefois, il est important de noter que le nouveau modèle ne signifie pas que la portée générale de la revue change, puisque MCBC a offert des publications dans ces domaines pendant plus de dix ans.

La revue maintiendra sa bonne réputation scientifique en continuant d'attribuer un rôle central aux rédacteurs scientifiques adjoints externes et aux pairs évaluateurs ainsi qu'au comité de rédaction, principalement composé de membres ne travaillant pas pour le gouvernement fédéral. Ces conseillers externes continueront d'éva- y Diffuser cet article sur Twitter

luer les manuscrits et de s'assurer que les articles publiés dans PSPMC demeurent de qualité élevée et enrichissent les connaissances canadiennes les plus récentes dans leur domaine.

Grâce au nouveau modèle de PSPMC, on passe également d'une diffusion passive des connaissances à un modèle plus intégré comprenant l'échange de connaissances en collaboration et en interaction. En effet, en matière de transfert de connaissances, les approches de diffusion traditionnelles (passives) mènent souvent à des résultats moins bons pour ce qui est de l'adoption des innovations en santé publique ${ }^{2}$. C'est pour tenir compte de ce contexte, ainsi que du contexte de transformation de l'ensemble de la gouvernance en sciences au sein de l'Agence de la santé publique du Canada (éditeur de la revue), que le nouveau modèle de gouvernance et d'édition de la revue a été proposé.

Jusqu'à présent, la santé publique se nourrissait de la création et de la publication de recherches appliquées. Or on ressent de plus en plus le besoin que ces connaissances soient mieux synthétisées et soit accessibles aux nombreux décideurs susceptibles de les utiliser ${ }^{3,4}$. Le renouveau de la revue PSPMC témoigne de l'ampleur et de la qualité des activités de collaboration scientifique, de surveillance et d'évaluation ou études réalisées par le gouvernement. La revue constitue une plateforme de diffusion d'envergure pour la science portant sur la promotion de la santé et sur la prévention des 
maladies chroniques de l'Agence de la santé publique du Canada. Notre but est de continuer à faire de la revue un véhicule très apprécié de communication et de soutien à la science et à la recherche comme à l'analyse et aux travaux connexes de collaboration évalués par les pairs dans le domaine de la santé publique, et ce, auprès d'un auditoire en recherche appliquée et en politiques et pratiques au Canada.

Dans le cadre de son objectif d'augmenter le volume de données probantes pertinentes sur les politiques et sur les interventions pouvant éclairer la prise de décision en politiques et pratiques, PSPMC a élargi l'éventail des types d'articles publiés pour y inclure des synthèses de données probantes et des communications courtes sur les données probantes, des études qualitatives et fondées sur des méthodes combinées, des études d'intervention ainsi qu'une section intitulée « Aperçu », qui permet d'obtenir de brefs comptes rendus statistiques des analyses de surveillance les plus récentes (voir http://www.phac-aspc.gc.ca/publicat/hpcdp -pspmc/authinfo-fra.php).

PSPMC démontre sa capacité à répondre à un besoin de mobilisation accrue en matière de mise en application et d'impact. Même si un sondage effectué en 2012 sur la satisfaction des intervenants a révélé que la plupart des répondants étaient satisfaits de la revue (taux de satisfaction globale de $90 \%$ ), certains ont fait remarquer que l'utilisation des médias sociaux pour promouvoir le contenu de la revue permettrait de mieux faire connaître celle-ci et d'en favoriser l'accès. Compte tenu de cela et des demandes pour un accès plus rapide aux données probantes, la revue devient mensuelle et entièrement en ligne, ce qui permet d'accélérer la fréquence et de réduire les délais de publication des articles. Nous allons promouvoir et partager les résultats publiés par l'intermédiaire des sites de réseaux sociaux professionnels, des webinaires et des plateformes de médias sociaux, et nous étudions également certaines options mobiles.
La revue mettra davantage l'accent sur la recherche et l'analyse collaboratives entre le gouvernement et les chercheurs externes, un éventail de praticiens en santé publique, des planificateurs de politiques de santé et des professionnels de domaines connexes. Dans le cadre de ce nouveau modèle, la revue fera un accueil particulièrement favorable aux articles issus d'une collaboration substantielle avec l'Agence de la santé publique du Canada ou Santé Canada en matière de corédaction (y compris avec le personnel des Instituts de recherche en santé du Canada) ou du fait d'un financement conjoint ou de l'utilisation de données conjointes de l'Agence de la santé publique ou de Santé Canada*.

Dans la même veine collaborative, PSPMC se renouvelle aussi pour augmenter l'accès à un éventail plus large de connaissances des systèmes communautaires et de santé publique et pour favoriser leur utilisation ${ }^{4}$. L'Agence sera en mesure de diffuser plus rapidement à l'externe les diverses synthèses de données probantes et les analyses canadiennes de grande qualité effectuées en collaboration avec d'autres parties prenantes : souvent, ces dernières ne sont pas publiées ailleurs sur le Web ni ne sont diffusées largement.

C'est avec plaisir que nous vous invitons à parcourir ce premier numéro du nouveau modèle de la revue. Les articles de recherche originaux - " Régime DASH et risque de cancer colorectal chez les adultes canadiens", de Jones-McLean et ses collaborateurs, et « Le syndrome de fatigue chronique et la fibromyalgie au Canada : prévalence et associations avec six indicateurs de l'état de santé », de Rusu et ses collaborateurs participent à l'enrichissement de la base de données probantes canadiennes dans ces domaines. Ce numéro offre également des synthèses des rapports de surveillance les plus récents de l'Agence, qui portent sur deux sujets importants : les indicateurs de santé périnatale et les anomalies congénitales. Enfin, vous êtes invités à jeter un coup d'œil à la section "Autres publications de l'ASPC », qui rassemble des articles collaboratifs évalués par les pairs et publiés ailleurs.

Nous espérons que vous aimerez plusieurs des spécificités de notre nouveau modèle de revue. Au nom de nos collègues de l'Agence de la santé publique du Canada, nous nous réjouissons à la perspective de continuer à collaborer avec vous à la création, à la synthèse et à la mobilisation de la recherche appliquée et de l'analyse, afin d'influer favorablement la promotion de la santé et la prévention des maladies chroniques au Canada.

\section{Références}

1. Clayton AJ. Éditorial - Rédacteur invité. Lancement d'un nouveau bulletin. Maladies chroniques au Canada. 1980;1(1):1.

2. Robinson K, Elliott SJ, Driedger SM et collab. Using linking systems to build capacity and enhance dissemination in heart health promotion: a Canadian multiple-case study. Health Educ Res. 2005;20(5):499-513.

3. Speller V, Wimbush E, Morgan A. Evidencebased health promotion practice: how to make it work? Promot Educ. 2005; Suppl 1: 15-20.

4. McDonald PW, Viehbeck S. From Evidencebased practice making to practice-based evidence making: creating communities of (research) and practice. Health Promot Pract. 2007;8(2):140-144.

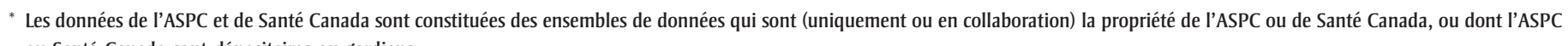
ou Santé Canada sont dépositaires ou gardiens. 